\title{
The Characterization of Fe Concretions in the Human Brain Exhibiting Neuro-degeneration with Brain Iron Accumulation (NBIA)
}

\author{
John McCarthy,* Susan Hayflick,** and Alex Buxbaum *** \\ *Portland State University, Materials and Manufacturing Research Instititue, Portland OR 97207 \\ ** Oregon Health and Science University, Dept. of Molecular \& Medical Genetics, Portland ,OR \\ 97239-3098 \\ *** FEI Company, Hillsboro, OR 97124-5793
}

NBIA is a genetic disease that shows high Fe content in concretions near blood vessels in the globus pallidus and widely distributed axonal swellings in the pallidonigral system. This pathology results in motor and cognitive problems that progressively worsen as the patient ages. [1,2] Optical imaging of stained brain tissue reveal these Fe rich concretions to have an ellipsoidal shape and likely to cluster near blood vessels. This study makes extensive use of SEM, Dual-Beam FIB and TEM to characterize these concretions. It is hoped that knowing what elements, compounds and structures are present in the concretions will help to understand the chain of reactions that create them and their role in neurodegneration.

The brain tissue available was prepared for optical imaging having been fixed and parafinized for preservation and then microtomed to $5 \mu \mathrm{m}$ thick sections. The section was deparafinized using solvents and rehydrated and stored in PBS prior to placing a portion on a coverslip and freeze drying at high vacuum followed by coating with $10 \mathrm{~nm}$ of evaporated Ti.

The freeze dried and coated specimen was then examined in an SEM equipped with an annular backscatter electron detector and a multi-window energy dispersive X-ray detector. Backscatter imaging was used to locate areas on the $1 \mathrm{~cm}$ square section of the brain where average $\mathrm{Z}$ was high compared to the low $\mathrm{Z}$ of the surrounding tissue. This was a rapid way to locate regions that were likely to be high in metals and follow this with X-ray microanalysis. The X-ray detector was used in the no window state to provide maximum sensitivity to low concentrations of metals and the light elements oxygen, nitrogen and carbon. This technique located regions high in $\mathrm{Ca}$ and $\mathrm{Fe}$. The Fe concretions are visible in figure 1 similar to the cluster of ellipsoids revealed by staining in optical imaging. In addition to Fe, X-ray microanalysis in the SEM detected high concentrations of O, $\mathrm{C}$ and $\mathrm{P}$ and some $\mathrm{Zn}$ in the concretions.

The SEM images allowed us to locate the 15 micron long cluster of concretions shown in figure 1 in a dual beam FIB equipped with a probe. The region of the cluster was then coated with Pt using the electron beam followed by a Ga beam deposited Pt bar, $1 \mu \mathrm{m} \times 1 \mu \mathrm{m} \times 15 \mu \mathrm{m}$, centered on the long axis of the cluster. A TEM cross-section was then excised from the cluster, attached to a grid, thinned to $150 \mathrm{~nm}$ thick and examined in a TEM/STEM. See figure 1. The higher resolution TEM images of the concretions revealed spherical structures $10-14 \mathrm{~nm}$ in diameter. Selected area electron diffraction produced 7 rings with all d-spacings matching hematite (rhombohedral $\mathrm{Fe}_{2} \mathrm{O}_{3}$, space group 167). See figure 2. Darkfield imaging using the first 2 rings in the pattern showed a high density of 1-5 nm crystallites within the concretions and X-ray microanalysis detected Fe and high concentrations of $\mathrm{O}$ and $\mathrm{C}$ in the TEM thin section. This indicates that the concretion contains protein, Fe oxide and 
ferritin at various stages of decomposition. Ferritin is the protein structure that stores and releases Fe in the body. Ferritin is a $12 \mathrm{~nm}$ protein shell with channels for the release of Fe that holds a hydrated Fe oxide, ferrihydrite $[\mathrm{FeO}(\mathrm{OH})]_{8}\left[\mathrm{FeO}\left(\mathrm{H}_{2} \mathrm{PO}_{4}\right)\right]$, at its center bonded to the protein shell by a phosphate group.

In addition to the detection of hematite, a cluster of faceted magnetite particles was also found in a portion of the $5 \mu \mathrm{m}$ thick freeze dried section placed on a TEM grid, located by backscatter imaging. Magnetite has a role in many neurological disorders. [3] An accumulation of biogenic magnetite can be a precursor to the appearance of clinical symptoms for Alzheimers disease patients. [4] [5]

\section{References}

[1] M. Davis, S. J. Hayflick and J. Jankovic, Movement Disorders 19(1) (2004)36.

[2] Y. M. Kuo, J. L. Duncan, S. J. Hayflick et. al. , Human Molecular Genetics 14(1) (2005)49.

[3] J. Dobson, Ann. NY Acad. Sci. 1012 (2004).

[4] D.Hautot, Q. A, Pankhurst, N.Kahn, et.al. , Proc.Royal Soc. B- Biology Letters 270(2003)S62-S64 [5] We acknowledge the NBIA Disorders Association for funding this work and FEI for access to the Nanoport Lab.
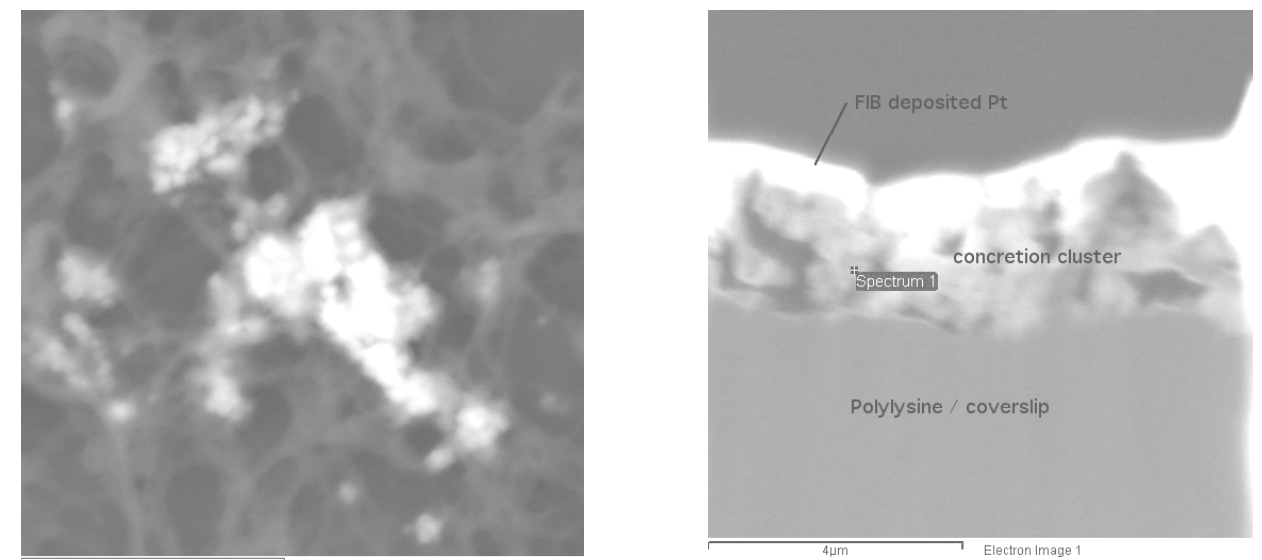

Figure 1. Backscatter electron 1mage of Fe-rich concretion cluster in freeze dried brain, left.

Marker 8 microns. Right, excised FIB section on the long axis of the cluster, $150 \mathrm{~nm}$ thick.

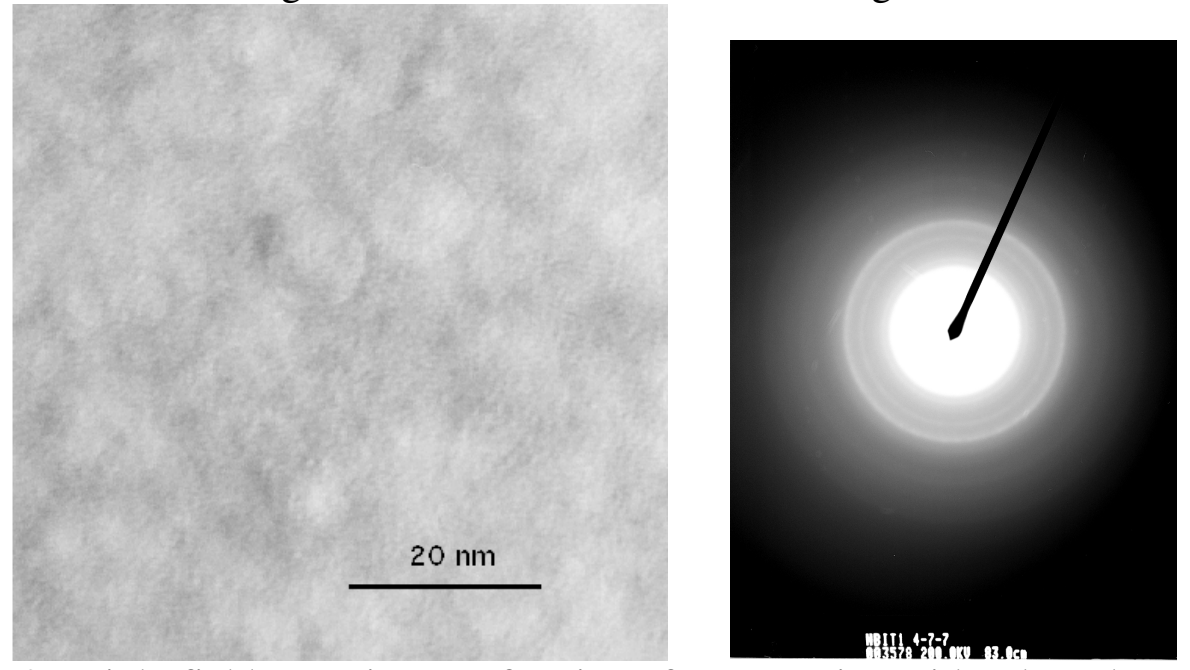

Figure 2. Bright field TEM image of region of a concretion with selected area electron diffraction. 